\title{
PROBABILISTIC MODEL OF REGGEON FIELD THEORY
}

\author{
K. G. BORESKOV \\ Institute of Theoretical and Experimental Physics, Moscow, Russia \\ E-mail: boreskov@heron.itep.ru
}

\begin{abstract}
The stochastic model of classical system of particles (partons), which dynamics includes random walk in plane as well as processes of death, splitting, annihilation and fusion of partons, is considered. A set of equations for multiparticle distribution functions for this system can be described in terms of diagrams of the Reggeon Field Theory (RFT) with supercritical pomeron, where rapidity plays a role of the time variable. The multiparticle inclusive distributions of partons correspond to multi-pomeron states in this analogy. In order to calculate the amplitude of hadron interaction at given energy we define an operator of linkage of any pair of partons from two parton sets (the projectile and the target). The form of the hadroninteraction (parton-linkage) operator is determined from the requirement for the result to be in correspondence to the RFT formulas. It is shown that the requirement of Lorentz invariance of the amplitude is fulfilled in this interpretation only at definite relation between the probabilities of parton splitting, annihilation and fusion. Interpretation of various methods of $s$-channel unitarization is discussed in terms of the model.
\end{abstract}

\section{Contents}

1 Introduction 3

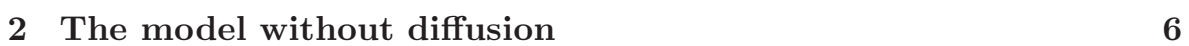

3 Account for spacial diffusion of partons 10

\begin{tabular}{|lll}
\hline 4 & Equivalence of the parton model to reggeon field theory & 16
\end{tabular}

$\begin{array}{lll}5 & \text { Interaction of colliding hadrons } & 18\end{array}$

$\begin{array}{lll}6 & \text { Examples } & 22\end{array}$

\begin{tabular}{lll}
\hline 7 & Unitarity & 27
\end{tabular}

$\begin{array}{ll}8 \text { Conclusions } & 29\end{array}$

$\begin{array}{ll}\text { Acknowledgments } & 30\end{array}$

References 30 
Yet say not sadly: they have left us! But say, with gratitude: they were." - Vasily Zhukovsky

This paper is dedicated to memory of Misha Marinov. One of the first papers published by Misha was a work done together with Roginsky on relation between $s$ - and $t$-channel helicity amplitudes which was important for description of spin particle interactions in Regge theory. I've just come to this field and was strongly impressed with mathematical elegance of this paper, especially with the fact that the direction to the unphysical point (centre-of-mass of $t$ channel) turned out to be real and physical. Unfortunately, this paper contained a minor mistake (change of helicity sign for transition from particle to antiparticle was not taken into account). I think that this mistake was related with Misha's style because it could be easily detected by consideration of the simplest example of $\pi N$ scattering. But Misha liked general considerations so much that didn't carry on elementary test. Later on these results were independently reproduced (without the mistake) in the well known paper by Trueman and Wick where examples were carefully considered.

In Theoretical Department Misha acted as a receptor providing an information about new physical ideas especially using advanced mathematical technique. He was in earnest about this mission and prepared these talks very carefully. He always had a detailed plan which he followed very hard and didn't allow uncontrolled discussions and digressions if they were not planned. Due to this hardness he always managed to convey to the audience main ideas and his own relation to them. Up to now my attitude to many of these topics is affected by primary Misha's interpretation.

Misha was born Teacher, and it was a mystery for me why he had no students in ITEP to teach or to supervise them. Once I asked him to give a couple of popular lectures on path integral for me and some colleagues. $\mathrm{He}$ agreed instantly, and as a result we got a half-year course of lectures, which was transformed eventually to the remarkable review in Physics Reports. And how many such opportunities we missed!

Everything what he did, Misha tried to execute professionally, either it was scientific paper writing, tea making, or karate training. I remember as during one of ITEP Winter Schools we were playing charades trying to show meaning of word or its part by performing short sketches. Misha chose to show just the word "charade" (in russian). First his team performed three sketches 
for its parts, and then, to show the word as a whole, they repeated all these sketches again at a rapid pace, representing the process of the game. It was well done, and we could see perfectionism of Misha as a theater director. To be more professional as an actor he used tooth-paste for his make-up (performing a role of devil).

Misha's unlimited erudition and knowledge were always at service of his colleagues. They consulted with him on any subject and always got thoughtful discussion and useful references, often to old and forgotten papers. The paper which I present in this volume just illustrates the lack of Misha's advices and consultations for me - when it has been finished I met the old paper by Grassberger and Sundermeyer where the same model for reggeon field theory was formulated ${ }^{2}$.

I liked to discuss with Misha Jewish history, origin of Jewish names, problems of Jewish young people at entrance examinations in universities, etc. But not less amount of exciting information I got from him on Russian history and language, literature, biology, and many other things (by the way, the origin of the word "eikonal" appearing in this paper and common with the word "icon" I knew from Misha).

Misha's high standards exerted influence on attitude to different situations, including ethical problems, in the circle of ITEP theorists. His opinion often was crucial giving, as a tuning fork, a point of reference. And now, what unphysical point Misha would be at, the orientation to his standards is still fixed for us.

\section{Introduction}

Processes of strong interactions at high energies are described usually in framework of reggeon theory 1 . Systematic description of high-energy interactions of hadrons in terms of reggeon field theory (RFT) was introduced by Gribov in ref.2. These results were obtained in a hybrid field theory model by calculation of Feynman diagrams containing the reggeon amplitudes as building blocks.

Experimental data on hadron interactions show that the total cross section is dominated by multiparticle processes and the number of secondaries increases with energy growth. Therefore only models taking into account large number of effective degrees of freedom (referred below as to partons for brevity) in a fast hadron can correspond to the nature of reggeon amplitudes.

Attempts to give the parton interpretation of the reggeon amplitudes started immediately after appearance of the notion of the reggeon. In ref.

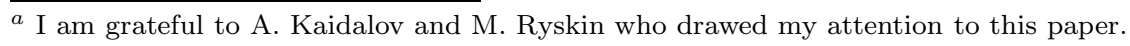


a representation of the pole reggeon diagram was introduced as a multiperipheral fluctuation of the fast hadron. Simultaneous interactions of several multiperipheral fluctuations with the target correspond to nonplanar reggeon diagrams and explain the presence of the regge-cut contributions 1 Later on interpretations of reggeon interactions were suggested using as inner degrees of freedom the objects of quantum chromodynamics - the quark-gluon string modele, the BFKL pomeron 6 , etc.

The common feature of all these models is consideration of a fast moving hadron as a complicated composite system which components (partons) are continuously distributed in rapidity values and randomly in the impact parameter plane. Hence, the Fock wave function of the fast hadron is a superposition of multiparton states and its structure depends on the hadron energy.

Interaction of the hadron with a target is realized only due to interactions of the most slow (in the target frame) partons produced step by step from faster components (short-range interaction in the rapidity space). The amplitude of interaction is determined by the number of slow partons $n(y)$ in the vicinity of the target. The probability of interaction with a target of only one of slow partons is proportional to $n(y)$ and corresponds to the regge-pole contribution. Interactions of two and more partons correspond to the regge cuts of second and higher orders. The cut contributions are sign-alternating because of shadowing effects.

Growth of hadron cross sections with energy gives evidences in favor of the so called supercritical pomeron $\left(\Delta_{P}=\alpha_{P}(0)-1>0\right)$. RFT with the supercritical pomeron encounters a number of difficulties which are not resolved in satisfactory way. The pole amplitude corresponding to the one-pomeron exchange increases with energy as $s^{\Delta}$ violating $s$-channel unitarity. Diagrams of higher orders, in principle, have to restore the unitarity. However, when the energy increases the number of essential diagrams grows drastically, and there is no satisfactory way to sum a series of reggeon diagrams at present. Usually one takes into account only a set of the simplest diagrams corresponding to the eikonal or "quasi-eikonal" approximation which corresponds to account for the non-enhanced cut only and disregards inter-pomeron interactions. The scattering amplitude in this approximation looks as for scattering on almost black disc of radius proportional to the rapidity $y$ in correspondence to the Froissart regime. In some approximations contributions of the enhanced regge cuts were summed. E.g. in the Schwimmer approximationt, which is justified for hadron-nucleus interactions, the fan-type enhanced diagrams are taken into account but reggeon loops are neglected. Analysis of more complicated sets of diagrams was carried out in refs 6 . 1 . However, the problem of construction of hadron scattering amplitude satisfying both $s$-channel and $t$-channel unitarity 
is not solved up to now.

In the parton picture the increase of the one-pomeron contribution with energy in the regime of the supercritical pomeron means that the number of soft partons grows exponentially with hadron rapidity, $n(y) \sim \exp (\Delta y)$. Thus, the mechanism of parton multiplication has to be present in the course of the rapidity evolution of the partonic fluctuation. It could be splitting of the multiperipheral ladder, gluon cascading, etc. From general arguments fusion of partons may also take place beside splitting.

The kinetic model of parton interactions equivalent to RFT was suggested in paper by Grassberger and Sundermeyer 11. In fact, this model gives a desription of stationary structure of the Fock wave function of a fast hadron.

In presented paper we discuss interaction of two parton systems in this model f . We analyze a possibility of consistent probabilistic interpretation of the interaction amplitude in this approach and, particularly, its Lorentz invariance.

The main assumption of the model is continuous parton evolution in rapidity, i.e. an origin of slower partons from the faster predecessors. In the course of evolution partons undergo a random walk in the impact parameter plane. Other essential ingredients of parton dynamics are processes of splitting of one parton into two ones and fusion of two partons into one parton. For generality we consider also vanishing ("death") one of partons at given rapidity and "annihilation" of pair of partons at close points. The correspondence can be proved between multiparticle parton distribution functions and multipomeron vertices of the RFT with lagrangian including the elementary three-pomeron coupling and the pomeron-scattering term. Thus, the pomeron in this model is not some specific object but just one-parton inclusive distribution, while many-pomeron states are connected to the inclusive multiparton distributions.

In order to introduce into the model hadron-hadron interactions it is necessary to define the operator of the interaction of two parton systems with different rapidities. We show here that this operator can be defined in the way maintaining the correspondence with the interaction amplitude given by summing all diagrams of RFT including loop diagrams. It is shown that if some definite relation between the constants of splitting, annihilation and fusion of partons holds, the hadron interaction amplitude depends only on the sum of rapidities of the interacting hadrons according to the Lorentz invariance requirement of the RFT. However the price for the correspondence with RFT is a loss of the probabilistic interpretation, because the interaction operator doesn't have, in general, a probabilistic meaning. Nevertheless, the interaction

${ }^{b}$ Unluckily, I found the paper 11 only after this text has been finished. I elaborated the same kinetic model for RFT so the content of first section overlap considerably with ref. 11 
amplitude does not violate $s$-channel unitarity, if initial parton distributions can be represented as superpositions of Poisson distributions.

The layout of the paper is as follows. In sect. 2 the model is formulated in simplified version which does not take into account parton diffusion in the transverse plane. In sect. 3 the diffusion of partons in the impact parameter plane is included and equations for the multiparticle distribution functions are derived both in coordinate and momentum representations. The equivalence of the equations for these multiparton distributions and equations for the many-pomeron vertices in the "cut" RFT is demonstrated in sect.4. In sect.5 we define the interaction operator for two parton sets and demonstrate independence of inelastic cross section on a choice of Lorentz frame. In sect.6 the main model approximations in RFT are considered - the eikonal approximation, the Schwimmer model and some others. The picture of an expanding black disc corresponding to the Froissart regime is discussed. The problem of $s$-channel unitarity is discussed in sect.7. Finally, in sect.8, we summarize the main results of the paper and discuss briefly possible generalizations and applications of this approach.

\section{The model without diffusion}

We discuss in this section the 0-dimensional model (without parton diffusion) which posesses some typical features of the general case. This approximation becomes adequate when it is possible to ignore parton diffusion, e.g. in the regime of the uniform density distribution or in the case of interactions with heavy nuclei.

Let us consider a system of partons with variable number of particles $N$, which evolves in time $y$ due to the following elementary processes: death of a parton, annihilation of two partons, splitting of any parton into two ones and fusion of arbitrary parton pair into one parton. Denote the probability of the $N$-parton state at the moment $y$ as $p_{N}(y)$, and the probabilities of death, annihilation, splitting and fusion of partons for the unit of time as $m_{1}, m_{2}, \lambda$ and $\nu$, respectively.

The evolution equations have the form:

$$
\begin{aligned}
& \frac{d p_{N}}{d y}=-\left(\lambda+m_{1}\right) N p_{N}-\left(\nu+m_{2}\right) N(N-1) p_{N}+\lambda(N-1) p_{N-1} \\
& \quad+m_{1}(N+1) p_{N+1}+\nu(N+1) N p_{N+1}+m_{2}(N+2)(N+1) p_{N+2},(N \geq 2), \\
& \frac{d p_{0}}{d y}=m_{1} p_{1}+2 m_{2} p_{2},
\end{aligned}
$$


$\frac{d p_{1}}{d y}=-\left(\lambda+m_{1}\right) p_{1}+2\left(m_{1}+\nu\right) p_{2}+6 m_{2} p_{3}$.

The initial condition for eqs (11) is given by the parton distribution at the moment $y=0$, and the conservation of the total probability $p(y)=\sum_{0}^{\infty} p_{N}(y)$ follows from (1):

$$
\frac{d p}{d y}=0
$$

The equivalent set of equations can be written down for the factorial moments $\mu_{s}$, defined as expectations of quantities $(N)_{s} \equiv \mathrm{N} ! /(\mathrm{N}-\mathrm{s}) !$ :

$$
\mu_{s}=\sum_{N=0}^{\infty}(N)_{s} p_{N} \equiv \sum_{N=0}^{\infty} \frac{N !}{(N-s) !} p_{N}, \quad(s=0,1,2, \ldots) .
$$

After multiplying the both sides of the eqs (1) to $(N)_{s}$ and expressing the products appearing in the right-hand sides in terms of $(N)_{s},(N)_{s-1}, \ldots$, we come to the set of equations for the moments:

$$
\begin{aligned}
\frac{d \mu_{s}}{d y}= & \lambda s(s-1) \mu_{s-1}+\left(\lambda-m_{1}\right) s \mu_{s} \\
& -\left(\nu+m_{2}\right) s(s-1) \mu_{s}-\left(\nu+2 m_{2}\right) s \mu_{s+1} .
\end{aligned}
$$

In particular, the equation (2) for $p(y) \equiv \mu_{0}(y)$ is reproduced at $s=0$.

It is convenient to define the generating function $G(w ; y)$, which is at the same time the exponential generating function for moments:

$$
G(w ; y)=\sum_{N=0}^{\infty} p_{N}(y) w^{N}=\sum_{s=0}^{\infty} \mu_{s}(y) \frac{(w-1)^{s}}{s !} .
$$

It satisfies the partial differential equation:

$$
\frac{\partial G}{\partial y}=(1-w)\left(m_{1}-\lambda w\right) \frac{\partial G}{\partial w}+(1-w)\left[m_{2}+\left(m_{2}+\nu\right) w\right] \frac{\partial^{2} G}{\partial w^{2}}
$$

with initial condition

$$
G(w ; 0) \equiv G_{0}(w)=\sum_{N=0}^{\infty} p_{N}(0) w^{N} .
$$

The boundary condition at $w=1$ is maintained automatically:

$$
G(1 ; y)=1 \text {. }
$$


8 K. G. Boreskov

In case $m_{1}=m_{2}=0$ it holds at $w=0$ :

$$
G(0 ; y)=G_{0}(0)
$$

Let us consider some special cases:

(a) The absence of parton fusion and annihilation $\left(\nu=0, m_{2}=0\right)$

In this case Eq.(5) becomes of 1 st order and after the change of variables $w \rightarrow \gamma$, where

$$
d \gamma=\frac{d w}{\lambda w^{2}-\left(\lambda+m_{1}\right) w+m_{1}}, \quad w(\gamma)=\frac{1-\left(m_{1} / \lambda\right) \exp \left[\left(\lambda-m_{1}\right) \gamma\right]}{1-\exp \left[\left(\lambda-m_{1}\right) \gamma\right]},
$$

one obtains

$$
\begin{aligned}
& \frac{\partial \hat{G}(\gamma ; y)}{\partial y}=\frac{\partial \hat{G}(\gamma ; y)}{\partial \gamma}, \\
& \hat{G}(\gamma ; 0)=\hat{G}_{0}(\gamma), \quad \hat{G}(-\infty ; y)=1
\end{aligned}
$$

with notation $\hat{G}(\gamma ; y) \equiv G(w(\gamma) ; y), \hat{G}_{0}(\gamma) \equiv G_{0}(w(\gamma))$. Its solution is a function of the sum of the variables $\gamma+y$ satisfying the initial condition (7),

$$
\hat{G}(\gamma ; y)=\hat{G}_{0}(\gamma+y)
$$

or, coming back to the variable $w$,

$$
G(y, w)=\sum_{N} W(y)^{N} p_{N}(0)=G_{0}(W(y))
$$

where

$$
\begin{aligned}
& W(y)=\frac{1-\eta e^{\Delta(\gamma+y)}}{1-e^{\Delta(\gamma+y)}}=\frac{w-\eta-\eta(w-1) e^{\Delta y}}{w-\eta-(w-1) e^{\Delta y}}, \\
& \Delta=\lambda-m_{1}, \quad \eta=m_{1} / \lambda .
\end{aligned}
$$

This distribution differs significantly from the Poisson distribution being characterized by a presence of strong correlations.

If there is $N_{0}$ particles in the initial state, then

$$
G(w ; y)=[W(y)]^{N_{0}}
$$


The mean value of particles increases exponentially with energy in this case:

$$
\mu_{1}(y)=N_{0} e^{\Delta y},
$$

and the second moment behaves as follows:

$$
\mu_{2}(y)=N_{0}\left(N_{0}+\frac{1+\eta}{1-\eta}\right) e^{2 \Delta y}-\frac{2 N_{0}}{1-\eta} e^{\Delta y} .
$$

In case when $m_{1} \neq 0$, the state without partons $(N=0)$ is so called absorbing state. The probability $p_{0}(y)$ can only increase:

$$
p_{0}(y)=\frac{\eta\left(e^{\Delta y}-1\right)}{e^{\Delta y}-\eta}
$$

If the process of parton death dominates $\left(\Delta=\lambda-m_{1}<0\right)$, then $p_{0}(y) \rightarrow 1$ at $y \rightarrow \infty$. If $\Delta>0$, this probability goes to the constant $\eta=m_{1} / \lambda$.

In the case of $m_{1}=0$ the probability $p_{0}(y) \equiv 0$, and the probability distribution can be written as

$$
p_{N}(y)=\left(\begin{array}{c}
N-1 \\
N-N_{0}
\end{array}\right) e^{-N_{0} \Delta y}\left(1-e^{-\Delta y}\right)^{N-N_{0}} .
$$

(b) The asymptotic in the fusion presence $(\nu \neq 0, y \rightarrow \infty)$

When $y \rightarrow \infty$ the state of the system approaches to the stationary state $G_{\infty}(w)=\lim _{y \rightarrow \infty} G(w ; y)$ of eq.(6) , which can be found from the equation for $\phi(w) \equiv d G_{\infty} / d w:$

$$
\left[m_{2}+w\left(m_{2}+\nu\right)\right] \frac{d \phi}{d w}+\left(m_{1}-\lambda w\right) \phi=0 .
$$

Solutions of this equation have the form

$$
\phi=C e^{a w}(w+c)^{-b},
$$

where $a=\lambda /\left(m_{2}+\nu\right), b=\left(m_{1} m_{2}+m_{1} \nu+m_{2} \lambda\right) /\left(m_{2}+\nu\right)^{2}, c=m_{2} /\left(m_{2}+\nu\right)$, and $C$ is determined from the initial condition. This gives

$$
G_{\infty}(w)=1+\int_{1}^{w} \phi(x) d x=1+C[F(w)-F(1)]
$$

with

$$
F(w)=\frac{e^{a(w+c)}}{a(w+c)^{b}}-(-a)^{b-1} b \Gamma(-b,-a(w+c)),
$$


where $\Gamma$ is the standard Gamma function of two arguments.

If $m_{1}=m_{2}=0$ we get

$$
G_{\infty}(w)=1-B+B e^{a(w-1)},
$$

with the constant $B$ determined by the initial condition.

Thus, the state of the system becomes asymptotically Poisson-like:

$$
\begin{aligned}
& \mu_{0} \underset{y \rightarrow \infty}{\longrightarrow} 1, \quad \mu_{s} \underset{y \rightarrow \infty}{\longrightarrow} B a^{s}, \quad s \geq 1, \\
& p_{0}(y)=1-B+B e^{-a}, \quad p_{N} \underset{y \rightarrow \infty}{\longrightarrow} B \frac{a^{N}}{N !} e^{-a}, \quad N \geq 1 .
\end{aligned}
$$

Though the moments $\mu_{s}$ depend on the subscript $s$ in power way the relations between moments reveal the correlation connected to independence of $p_{0}$ from other states:

$$
\mu_{1}(\infty)=B a, \quad \mu_{s}(\infty)=B a^{s} \neq\left[\mu_{1}(\infty)\right]^{s} .
$$

At $B=1$ one gets the purely Poisson distribution and all correlations vanish.

The value of the constant $B$ which determines the asymptotical behaviour depends on a choice of the initial condition. Due to (9)

$$
B=\frac{1-G_{0}(0)}{1-e^{-a}} .
$$

Note that $G_{0}(0)=p_{0}(0)$ and if $p_{0}(0)=p_{0}(y)=0$, then $B=\left(1-e^{-a}\right)^{-1}$.

If $m_{1}$ (or $m_{2}$ ) differs from zero the state with $N=0$ is the absorbing one. The death or annihilation processes dominate asymptotically in this case and, eventually, the system goes to the state without particles:

$$
p_{N} \underset{y \rightarrow \infty}{\longrightarrow} \delta_{N 0}, \quad G_{\infty}(w)=1 .
$$

This conclusion can be done from direct analysis of the kinetic equations (11), after putting to zero the derivatives in the left-hand sides.

Note that if $a=\lambda / \nu \gg 1$, the average number of particles in the system is large and its state can be described preasymptotically in thermodynamical approximation by eq.(26), though eventually at very large $y$ it goes to the absorbing state.

\section{Account for spacial diffusion of partons}

In this section we take into account a possibility of random walk of partons in the transverse plane in the course of evolution. We assume that a transverse coordinate of a parton, $\mathbf{b}_{i}$, gets for an infinitely small interval $d y$ an 
(isotropic) increment $d \mathbf{b}_{i}$ such that $\left(d \mathbf{b}_{i}\right)^{2}=D d y$. Note that this assumption on the character of the random walk of partons results in the linear form of the pomeron trajectory. It is a hypothesis only and other dynamical models for parton diffusion are conceivable 9

Let us formulate now dynamical equations for the parton system. Denote the probability for the system to be in the state with $N$ partons at the moment $y$ as $p_{N}(y)$, and the parton distribution in the impact plane as $\rho_{N}\left(y ; \mathbf{b}_{1}, \ldots, \mathbf{b}_{N}\right)$. To simplify the notations we shall omit the argument $y$ often, and denote the set of $N$ transverse coordinates as $\mathcal{B}_{N} \equiv\left\{\mathbf{b}_{1}, \ldots, \mathbf{b}_{N}\right\}$. All partons are supposed to be of the same type 9 , and $\rho_{N}$ is a symmetric function of coordinates normalized as

$$
\frac{1}{N !} \int d \mathcal{B}_{N} \rho_{N}\left(y ; \mathcal{B}_{N}\right)=p_{N}(y)
$$

where $d \mathcal{B}_{N}=d \mathbf{b}_{1} \ldots d \mathbf{b}_{N}$, and $p_{N}(y)$ is the probability to have the $N$-parton state at the moment $y$.

The following changes of the state of the system for an interval $d y$ are possible: change of position of any parton to $\left(d \mathbf{b}_{i}\right)^{2}=D d y$; death of a parton with the probability $m_{1} d y$; splitting of one parton into two ones with same coordinates with the probability $\lambda d y$, and, for two partons sufficiently close one to another, processes of their annihilation with vanishing both partons and their fusion into one parton. Assuming that the parton size is small enough (compared to interparton distances) the annihilation and fusion probability of partons at points $\mathbf{b}_{k}$ and $\mathbf{b}_{l}$ can be described as $m_{2} \delta\left(\mathbf{b}_{k}-\mathbf{b}_{l}\right)$ and $\nu \delta\left(\mathbf{b}_{k}-\mathbf{b}_{l}\right)$ correspondingly . Let us stress that the constants $m_{2}$ and $\nu$ are dimensional in contrast to the situation of sect.2.

Thus, the evolution equations for the densities $\rho_{N}$ have the following form:

$$
\begin{aligned}
& \frac{d \rho_{N}\left(y ; \mathcal{B}_{N}\right)}{d y}=D \nabla_{N}^{2} \rho_{N}\left(y ; \mathcal{B}_{N}\right)-\left(m_{1}+\lambda\right) N \rho_{N}\left(y ; \mathcal{B}_{N}\right) \\
& +m_{1} \int d b_{N+1} \rho_{N+1}\left(y ; \mathcal{B}_{N}, \mathbf{b}_{N+1}\right)+\lambda \sum_{k, l=1}^{N \geqslant 2} \rho_{N-1}\left(y ; \mathcal{B}_{N}^{(l)}\right) \delta\left(\mathbf{b}_{k}-\mathbf{b}_{l}\right)
\end{aligned}
$$

$\bar{c}$ One can consider a discrete model of parton diffusion by granulation the transverse space into small cells. In this case the random walk is included to the kinetic scheme on equal footing with splitting and fusion. The size of a cell plays the role of a cut-off for the model. ${ }^{d}$ If the internal quantum numbers of partons are introduced then relations between the constants of the RFT corresponding to given parton model, particularly between the intercept and the triple pomeron coupling, will be changed.

$e$ As it was mentioned, one can consider parton coordinates as discrete quantities in order to regularize divergences of the model. This results in the correspondingly smoothed $\delta$ functions. 
12 K. G. Boreskov

$$
\begin{aligned}
& -\left(m_{2}+\nu\right) \sum_{k, l=1}^{N \geqslant 2} \rho_{N}\left(y ; \mathcal{B}_{N}\right) \delta\left(\mathbf{b}_{k}-\mathbf{b}_{l}\right)+m_{2} \int d \mathbf{b}_{N+1} \rho_{N+2}\left(y ; \mathcal{B}_{N}, \mathbf{b}_{N+1}, \mathbf{b}_{N+1}\right) \\
& +\nu \int d \mathbf{b}_{N+1} \rho_{N+1}\left(y ; \mathcal{B}_{N}, \mathbf{b}_{N+1}\right) \sum_{k=1}^{N} \delta\left(\mathbf{b}_{k}-\mathbf{b}_{N+1}\right)
\end{aligned}
$$

where $\nabla_{N}^{2}=\sum_{i=1}^{N}\left(\partial_{\alpha}^{2} \rho_{N} / \partial z_{i \alpha}^{2}\right), \alpha=1,2$, and $\mathcal{B}_{N}^{(l)}$ means the set $\mathcal{B}_{N}$, from which the coordinate $\mathbf{b}_{l}$ is removed $\left(\mathcal{B}_{N}^{(l)}=\mathcal{B}_{N} \backslash \mathbf{b}_{l}\right)$.

One can also define the Fourier transformed distributions

$$
\sigma_{N}\left(y ; \mathcal{K}_{N}\right)=\int d \mathcal{B}_{N} \rho_{N}\left(y ; \mathcal{B}_{N}\right) e^{i \mathbf{k}_{1} \mathbf{b}_{1}+\cdots+i \mathbf{k}_{N} \mathbf{b}_{N}}
$$

where $\mathcal{K}_{N}=\left\{\mathbf{k}_{1}, \ldots, \mathbf{k}_{N}\right\}$, with normalization

$$
\sigma_{N}(y ; 0, \ldots, 0)=N ! p_{N}(y) .
$$

In the momentum representation the evolution equations take the form:

$$
\begin{aligned}
& \frac{d \sigma_{N}\left(y ; \mathcal{K}_{N}\right)}{d y}=-D\left(\sum_{i=1}^{N} \mathbf{k}_{i}^{2}\right) \sigma_{N}\left(y ; \mathcal{K}_{N}\right)-\left(m_{1}+\lambda\right) N \sigma_{N}\left(y ; \mathcal{K}_{N}\right) \\
& \quad+m_{1} \sigma_{N+1}\left(y ; \mathcal{K}_{N}, 0\right)+\lambda \sum_{k, l=1}^{N \geqslant 2} \sigma_{N-1}\left(y ; \mathcal{K}_{N}^{(k l)}, \mathbf{k}_{k}+\mathbf{k}_{l}\right) \\
& \quad-\left(m_{2}+\nu\right) \int \frac{d \mathbf{q}}{(2 \pi)^{2}} \sum_{k, l=1}^{N \geqslant 2} \sigma_{N}\left(y ; \mathcal{K}_{N}^{(k l)}, \mathbf{k}_{k}+\mathbf{q}, \mathbf{k}_{l}-\mathbf{q}\right) \\
& \quad+m_{2} \int \frac{d \mathbf{q}}{(2 \pi)^{2}} \sigma_{N+2}\left(y ; \mathcal{K}_{N}, \mathbf{q},-\mathbf{q}\right) \\
& \quad+\nu \int \frac{d \mathbf{q}}{(2 \pi)^{2}} \sum_{k=1}^{N} \sigma_{N+1}\left(y ; \mathcal{K}_{N}^{(k)}, \mathbf{k}_{k}+\mathbf{q},-\mathbf{q}\right)
\end{aligned}
$$

where $\mathcal{K}_{N}^{(k)}=\mathcal{K}_{N} \backslash \mathbf{k}_{k}$ and $\mathcal{K}_{N}^{(k l)}=\mathcal{K}_{N} \backslash\left\{\mathbf{k}_{k}, \mathbf{k}_{l}\right\}$ are the sets of momenta $\mathcal{K}_{N}$ with the momentum $\mathbf{k}_{k}$ or, respectively, the momenta $\mathbf{k}_{k}$ and $\mathbf{k}_{l}$, removed.

It is convenient, in the spirit of statistical mechanics, to introduce instead of distributions $\rho_{N}\left(y, \mathcal{B}_{N}\right)$ a set of the multiparticle distributions $f_{s}^{(N)}\left(y ; z_{1}, \ldots z_{s}\right), s=1, \ldots, N$, which correspond to fixation of the coordinates of $s$ partons and integration over coordinates of all rest:

$$
f_{s}^{(N)}\left(y ; \mathcal{Z}_{s}\right)=\frac{1}{N !} \int d \mathcal{B}_{N} \rho_{N}\left(y ; \mathcal{B}_{N}\right) A_{s}^{(N)}\left(\mathcal{B}_{N} \mid \mathcal{Z}_{s}\right)
$$


where, as above, $\mathcal{Z}_{s}=\left\{\mathbf{z}_{1}, \ldots, \mathbf{z}_{s}\right\}$. Here the function

$$
A_{s}^{(N)}\left(\mathcal{B}_{N} \mid \mathcal{Z}_{s}\right)=\sum_{\mathcal{I}_{s}^{(N)}} \delta\left(\mathbf{z}_{1}-\mathbf{b}_{i_{1}}\right) \delta\left(\mathbf{z}_{2}-\mathbf{b}_{i_{2}}\right) \ldots \delta\left(\mathbf{z}_{s}-\mathbf{b}_{i_{s}}\right),
$$

where summation is taken over all sets $\mathcal{I}_{s}^{(N)}$ of noncoincident indices $i_{1}, i_{2} \ldots i_{s}$ from the set $\{1,2, \ldots, N\}$, is a sum of $\delta$-function terms which fix coordinates of $s$ partons from $N$ ones.

The total multiparticle distributions are defined by summation over states with different number of partons:

$$
f_{s}\left(y ; \mathcal{Z}_{s}\right)=\sum_{N} f_{s}^{(N)}\left(y ; \mathcal{Z}_{s}\right)
$$

The normalization of the functions $f_{s}^{(N)}\left(y ; \mathcal{Z}_{s}\right)$ follows from eq.(29):

$$
\int d \mathcal{Z}_{s} f_{s}^{(N)}\left(y ; \mathcal{Z}_{s}\right)=(N)_{s} p_{N}
$$

where, as above, $(N)_{s}=N ! /(N-s)$ ! . Correspondingly, the distribution $f_{s}\left(y ; \mathcal{Z}_{s}\right)$ is normalized to the mean factorial moment:

$$
\int d \mathcal{Z}_{s} f_{s}\left(y ; \mathcal{Z}_{s}\right)=<(N)_{s}>\equiv \mu_{s}(y)
$$

The functions $f_{s}^{(N)}\left(y ; \mathcal{Z}_{s}\right)$ are related by the reduction equation:

$$
f_{s-r}^{(N)}\left(y ; \mathcal{Z}_{s-r}\right)=\frac{(N-s) !}{(N-r) !} \int d z_{r+1} \ldots d z_{s} f_{s}^{(N)}\left(y ; \mathcal{Z}_{s}\right) .
$$

Quite similarly, one can introduce the momentum multiparticle distributions $g_{s}^{(N)}\left(y ; \mathcal{Q}_{s}\right)$, connected to the functions $f_{s}^{(N)}$ with Fourier transform:

$$
\begin{aligned}
& g_{s}^{(N)}\left(y ; \mathcal{Q}_{s}\right)=\int d \mathcal{Z}_{s} e^{i \mathbf{q}_{1} \mathbf{z}_{1}} \ldots e^{i \mathbf{q}_{s} \mathbf{z}_{s}} f_{s}^{(N)}\left(y ; \mathcal{Z}_{s}\right) \\
& g_{s}\left(y ; \mathcal{Q}_{s}\right)=\sum_{N} g_{s}^{(N)}\left(y ; \mathcal{Q}_{s}\right)
\end{aligned}
$$

These functions are obtained from the distributions $\sigma_{N}\left(y ; \mathcal{K}_{N}\right)$, if to put $s$ momenta equaled to $q_{1}, \ldots, q_{s}$, and all the rest - to zeros:

$$
g_{s}^{(N)}\left(y ; \mathcal{Q}_{s}\right)=\frac{1}{(N-s) !} \sigma_{N}\left(q_{1}, \ldots, q_{s}, 0, \ldots, 0\right) .
$$


14 K. G. Boreskov

The reduction relation has the form:

$$
g_{s-r}^{(N)}\left(y ; \mathcal{Q}_{s-r}\right)=\frac{(N-s) !}{(N-r) !} g_{s}^{(N)}(y ; \mathcal{Q}_{s-r}, \underbrace{0, \ldots, 0}_{r \text { times }}),
$$

and normalization looks as

$$
\begin{aligned}
& g_{s}^{(N)}(y ; 0, \ldots, 0)=(N){ }_{s} p_{N} \\
& g_{s}(y ; 0, \ldots, 0)=\mu_{s}(y)
\end{aligned}
$$

The simplest way to get the kinetic equations for multiparticle distributions is to reduce eq.(33) by means of eq.(42) from the function $g_{N}^{(N)} \equiv \sigma_{N}$ to the function $g_{s}^{(N)}$. This gives

$$
\begin{aligned}
& \frac{d}{d y} g_{s}^{(N)}\left(y ; \mathcal{Q}_{s}\right)=-D\left(\sum_{a=1}^{s} \mathbf{q}_{a}^{2}\right) g_{s}^{(N)}\left(y ; \mathcal{Q}_{s}\right)-\left(m_{1}+\lambda\right) N g_{s}^{(N)}\left(y ; \mathcal{Q}_{s}\right) \\
& \quad+m_{1}(N-s+1) g_{s}^{(N+1)}\left(y ; \mathcal{Q}_{s}\right)+\lambda(N+s-1) g_{s}^{(N-1)}\left(y ; \mathcal{Q}_{s}\right) \\
& \quad+\lambda \sum_{k, l=1}^{s \geqslant 2} g_{s-1}^{(N-1)}\left(y ; \mathcal{Q}_{s}^{(k, l)}, \mathbf{q}_{k}+\mathbf{q}_{l}\right) \\
& \quad-\left(m_{2}+\nu\right) \int \frac{d \mathbf{q}}{(2 \pi)^{2}} \sum_{k, l=1}^{s \geqslant 2} g_{s}^{(N)}\left(y ; \mathcal{Q}_{s}^{(k, l)}, \mathbf{q}_{k}+\mathbf{q}, \mathbf{q}_{l}-\mathbf{q}\right) \\
& \quad-2\left(m_{2}+\nu\right) \int \frac{d \mathbf{q}}{(2 \pi)^{2}} \sum_{k=1}^{s} g_{s+1}^{(N)}\left(y ; \mathcal{Q}_{s}^{(k)}, \mathbf{q}_{k}+\mathbf{q},-\mathbf{q}\right) \\
& \quad-\left(m_{2}+\nu\right) \int \frac{d \mathbf{q}}{(2 \pi)^{2}} g_{s+2}^{(N)}\left(y ; \mathcal{Q}_{s}, \mathbf{q},-\mathbf{q}\right)+m_{2} \int \frac{d \mathbf{q}}{(2 \pi)^{2}} g_{s+2}^{(N+2)}\left(y ; \mathcal{Q}_{s}, \mathbf{q},-\mathbf{q}\right) \\
& \quad+\nu \int \frac{d \mathbf{q}}{(2 \pi)^{2}} \sum_{k=1}^{s} g_{s+1}^{(N+1)}\left(y ; \mathcal{Q}_{s}^{(k)}, \mathbf{q}_{k}+\mathbf{q},-\mathbf{q}\right) \\
& \quad+\nu \int \frac{d \mathbf{q}}{(2 \pi)^{2}} g_{s+2}^{(N+1)}\left(y ; \mathcal{Q}_{s}, \mathbf{q},-\mathbf{q}\right) .
\end{aligned}
$$

Note here, that when doing the reduction one has to consider separately the cases when summation variables $i, k$ are less and greater than $s$, and this increases a number of terms with coefficients $\lambda$ and $\nu$. The combinatorial coefficients appear in the equation due to this consideration (the common factor $(N-s)$ ! is omitted in the equation). 
Part of terms cancel after summation over $N$ (particularly terms containing $g_{s+2}$ ) and one gets

$$
\begin{aligned}
& \frac{d}{d y} g_{s}\left(y ; \mathcal{Q}_{s}\right)=-D\left(\sum_{a=1}^{s} \mathbf{q}_{a}^{2}\right) g_{s}\left(y ; \mathcal{Q}_{s}\right)+\left(\lambda-m_{1}\right) s g_{s}\left(y ; \mathcal{Q}_{s}\right) \\
& \quad+\lambda \sum_{k, l=1}^{s \geqslant 2} g_{s-1}\left(y ; \mathcal{Q}_{s}^{(k, l)}, \mathbf{q}_{k}+\mathbf{q}_{l}\right)-\nu \int \frac{d \mathbf{q}}{(2 \pi)^{2}} \sum_{k, l=1}^{s \geqslant 2} g_{s}\left(y ; \mathcal{Q}_{s}^{(k, l)}, \mathbf{q}_{k}+\mathbf{q}, \mathbf{q}_{l}-\mathbf{q}\right) \\
& \quad-\left(2 m_{2}+\nu\right) \int \frac{d \mathbf{q}}{(2 \pi)^{2}} \sum_{k=1}^{s} g_{s+1}\left(y ; \mathcal{Q}_{s}^{(k)}, \mathbf{q}_{k}+\mathbf{q},-\mathbf{q}\right), \quad(s=1,2, \ldots) . \quad(45)
\end{aligned}
$$

The equation for $f_{s}\left(y ; \mathcal{Z}_{s}\right)$ can be obtained both by Fourier transform of (45) or directly from the evolution equation (30) for $\rho_{N}$ using the definition (34) (it is necessary again to account for accurate combinatorics for cases $i, k \leq s$ and $i, k>s)$. As a result one gets the following equations for $f_{s}^{(N)}$ :

$$
\begin{aligned}
& \frac{d}{d y} f_{s}^{(N)}\left(y ; \mathcal{Z}_{s}\right)=D \vec{\nabla}_{s}^{2} f_{s}^{(N)}\left(y ; \mathcal{Z}_{s}\right)-\left(m_{1}+\lambda\right) N f_{s}^{(N)}\left(y ; \mathcal{Z}_{s}\right) \\
& \quad+m_{1}(N-s+1) f_{s}^{(N+1)}\left(y ; \mathcal{Z}_{s}\right)+\lambda(N-1+s) f_{s}^{(N-1)}\left(y ; \mathcal{Z}_{s}\right) \\
& \quad+\lambda \sum_{k, l=1}^{s \geqslant 2} f_{s-1}^{N-1}\left(y ; \mathcal{Z}_{s}^{(l)}\right) \delta\left(\mathbf{z}_{k}-\mathbf{z}_{l}\right)-\left(m_{2}+\nu\right) \sum_{k, l=1}^{s \geqslant 2} f_{s}^{(N)}\left(y ; \mathcal{Z}_{s}\right) \delta\left(\mathbf{z}_{k}-\mathbf{z}_{l}\right) \\
& \quad-2\left(m_{2}+\nu\right) \sum_{k=1}^{s} f_{s+1}^{(N)}\left(y ; \mathcal{Z}_{s}^{(k)}, \mathbf{z}_{k}, \mathbf{z}_{k}\right)-\left(m_{2}+\nu\right) \int d \mathbf{u} f_{s+2}^{(N)}\left(y ; \mathcal{Z}_{s}, \mathbf{u}, \mathbf{u}\right) \\
& \quad+m_{2} \int d \mathbf{u} f_{s+2}^{(N+2)}\left(y ; \mathcal{Z}_{s}, \mathbf{u}, \mathbf{u}\right)+\nu \sum_{k=1}^{s} f_{s+1}^{(N+1)}\left(y ; \mathcal{Z}_{s}^{(k)}, \mathbf{z}_{k}, \mathbf{z}_{k}\right) \\
& \quad+\nu \int d \mathbf{u} f_{s+2}^{(N+1)}\left(y ; \mathcal{Z}_{s}, \mathbf{u}, \mathbf{u}\right)
\end{aligned}
$$

and the set of equations for the total distribution functions $f_{s}\left(y ; \mathcal{Z}_{s}\right)$ :

$$
\begin{aligned}
& \frac{d}{d y} f_{s}\left(y ; \mathcal{Z}_{s}\right)=D \vec{\nabla}_{s}^{2} f_{s}\left(y ; \mathcal{Z}_{s}\right)+\left(\lambda-m_{1}\right) s f_{s}\left(y ; \mathcal{Z}_{s}\right)+ \\
& \quad+\lambda \sum_{k, l=1}^{s \geqslant 2} f_{s-1}\left(y ; \mathcal{Z}_{s}^{(l)}\right) \delta\left(\mathbf{z}_{k}-\mathbf{z}_{l}\right)-\nu \sum_{k, l=1}^{s \geqslant 2} f_{s}\left(y ; \mathcal{Z}_{s}\right) \delta\left(\mathbf{z}_{k}-\mathbf{z}_{l}\right)- \\
& \quad-\left(2 m_{2}+\nu\right) \sum_{k=1}^{s} f_{s+1}\left(y ; \mathcal{Z}_{s}^{(k)}, \mathbf{z}_{k}, \mathbf{z}_{k}\right), \quad(s=1,2, \ldots)
\end{aligned}
$$


Thus, we came to equations for the sets of the functions $f_{s}\left(y ; \mathcal{Z}_{s}\right)$ or $g_{s}\left(y ; \mathcal{Q}_{s}\right)$ which contain only distributions averaged over states with various numbers of partons.

\section{Equivalence of the parton model to reggeon field theory}

It will be shown in this section that eqs (45), (47) are equivalent to equations for reggeon vertices of the reggeon field theory with a particular set of coupling constants - the reggeon intercept $\Delta=\lambda-m_{1}$, the trajectory slope $\alpha^{\prime}=D$, the constant of splitting of one pomeron into two ones $\lambda$, the constant of fusion of two pomerons into one $2 m_{2}+\nu$ and the constant of pomeron scattering equaled to $\nu$.

The simplest way to see this, is to make the Mellin transform of the function $g_{s}(y)$ :

$$
G_{s}\left(\omega ; \mathcal{Q}_{s}\right)=\int_{0}^{\infty} d y e^{-\omega y} g_{s}\left(y ; \mathcal{Q}_{s}\right), g_{s}\left(y, \mathcal{Q}_{s}\right)=\frac{1}{2 \pi i} \int_{\uparrow} d \omega e^{\omega y} G_{s}\left(\omega ; \mathcal{Q}_{s}\right)
$$

where the integration in second formula is carried out in the complex plane $\omega$ along the infinite contour parallel to the imaginary axis.

One gets the following set of equations for the functions $G_{s}(\omega)$

$$
\begin{aligned}
& \omega G_{s}\left(\omega ; \mathcal{Q}_{s}\right)=D\left(\sum_{a=1}^{s} q_{a}^{2}\right) G_{s}\left(\omega ; \mathcal{Q}_{s}\right)+\left(\lambda-m_{1}\right) s G_{s}\left(\omega ; \mathcal{Q}_{s}\right) \\
& +\lambda \sum_{k, l=1}^{s} G_{s-1}\left(\omega ; \mathcal{Q}_{s}^{(k l)}, \mathbf{q}_{k}+\mathbf{q}_{l}\right)-\nu \int \frac{d \mathbf{q}}{(2 \pi)^{2}} \sum_{k, l=1}^{s} G_{s}\left(\omega ; \mathcal{Q}_{s}^{(k l)}, \mathbf{q}_{k}+\mathbf{q}, \mathbf{q}_{l}-\mathbf{q}\right) \\
& \quad-\left(2 m_{2}+\nu\right) \int \frac{d \mathbf{q}}{(2 \pi)^{2}} \sum_{k=1}^{s} G_{s+1}\left(\omega ; \mathcal{Q}_{s}^{(k)}, \mathbf{q}_{k}+\mathbf{q},-\mathbf{q}\right) .
\end{aligned}
$$

Let the initial condition for (45) be

$$
g_{s}\left(y=0 ; \mathcal{Q}_{s}\right)=\delta_{s s_{0}} .
$$

Then $g_{s}\left(y ; \mathcal{Q}_{s}\right)$ (respectively $G_{s}\left(\omega ; \mathcal{Q}_{s}\right)$ in $\omega$ representation) have meaning of vertices of transition of $s_{0}$ reggeons into $s$ reggeons. The function of free propagation (diffusion without splitting, annihilation and fusion) of $s$ reggeons in the $\omega$ representation has the form

$$
D_{s}^{(0)}\left(\omega ; \mathcal{Q}_{s}\right)=\left(\omega-D \sum_{a=1}^{s} q_{a}^{2}\right)^{-1}
$$


and the renormalized propagator can be defined as

$$
D_{s}\left(\omega ; \mathcal{Q}_{s}\right)=\left(\omega-s\left(\lambda-m_{1}\right)-D \sum_{a=1}^{s} q_{a}^{2}\right)^{-1} .
$$

If define the reduced vertices

$$
\Gamma_{s}^{s_{0}}\left(\omega ; \mathcal{Q}_{s}\right)=\left[D_{s}\left(\omega ; \mathcal{Q}_{s}\right)\right]^{-1} G_{s}\left(\omega ; \mathcal{Q}_{s}\right),
$$

the equations (49) take the form

$$
\begin{aligned}
& \Gamma_{s}^{s_{0}}\left(\omega ; \mathcal{Q}_{s}\right)=\lambda \sum_{k, l=1}^{s} \Gamma_{s-1}^{s_{0}}\left(\omega ; \mathcal{Q}_{s}^{(k l)}, \mathbf{q}_{k}+\mathbf{q}_{l}\right) D_{s-1}\left(\omega ; \mathcal{Q}_{s}\right)- \\
& -\nu \int \frac{d \mathbf{q}}{(2 \pi)^{2}} \sum_{k, l=1}^{s} \Gamma_{s}^{s_{0}}\left(\omega ; \mathcal{Q}_{s}^{(k l)}, \mathbf{q}_{k}+\mathbf{q}, \mathbf{q}_{l}-\mathbf{q}\right) D_{s}\left(\omega ; \mathcal{Q}_{s}\right)- \\
& \quad-\left(2 m_{2}+\nu\right) \int \frac{d \mathbf{q}}{(2 \pi)^{2}} \sum_{k=1}^{s} \Gamma_{s+1}^{s_{0}}\left(\omega ; \mathcal{Q}_{s}^{(k)}, \mathbf{q}_{k}+\mathbf{q},-\mathbf{q}\right) D_{s+1}\left(\omega ; \mathcal{Q}_{s}\right)
\end{aligned}
$$

which corresponds to the rules of reggeon diagram techniquel and is described by diagrams of fig. 1 .

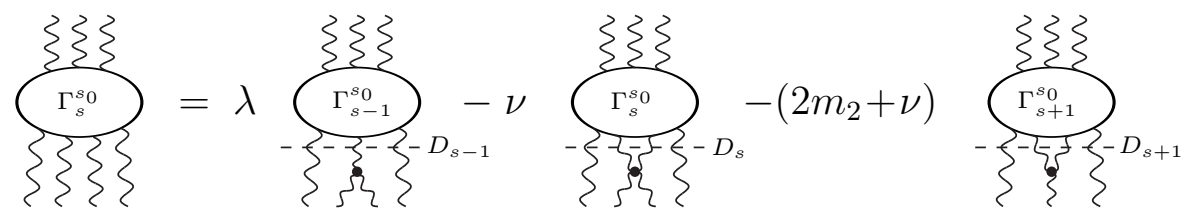

Figure 1: Diagrammatic representation of equations for the inclusive parton distributions which reproduces diagrams of RFT

Another correspondence of eqs (54) with reggeon theory is their treatment as equations of "inelastic" reggeon theory induced by Abramovsky-GribovKancheli cutting rules 12 (see details in ref. 13 ). Suppose that the contribution of some reggeon graph $\mathcal{G}$ to the scattering amplitude is schematically represented as $-i \hat{T}^{(\mathcal{G})}\left(\left\{-D_{k}\right\}\right)$, i.e. depends on the set of reggeon propagators $\left\{D\left(y_{k}, \mathbf{q}_{k}\right)\right\}$ integrated with required weights. Its contribution to the total cross section is $\sigma^{\text {tot }(\mathcal{G})} / 2=\operatorname{Im}\left[-i \hat{T}^{(\mathcal{G})}\left(\left\{-D_{k}\right\}\right)\right]$. Then (see ref. 13$)$ the inelastic discontinuity of this diagram, i.e. its contribution to the inelastic cross section, can be written as $\sigma^{\operatorname{inel}(\mathcal{G})}=-\hat{T}^{\mathcal{G}}\left(\left\{-2 \operatorname{Im}\left(i D_{k}\right)\right\}\right)$, that is it also can be calculated 
by means of reggeon theory but with cut propagators. Contributions of the diagram $\mathcal{G}$ to generating functions for different inelastic characteristics also can be determined from this theory 13 by changing arguments of operator $\hat{T}^{\mathcal{G}}$.

Thus, evolution of the parton distributions in rapidity is described by diagrams of reggeon field theory. The pomerons do not come out in this description as some individual propagating objects. The evolution in rapidity of the $s$-pomeron state corresponds to the developing in time the $s$-particle inclusive distribution of partons (i.e. when momenta of $s$ partons are fixed and momenta of all others are arbitrary).

\section{Interaction of colliding hadrons}

In previous sections the parton model was formulated which dynamics is equivalent to reggeon field theory. Parton distributions at different $y$ given by this model can be associated with the stationary structure of parton distributions in rapidity for a fast moving hadron (i.e. with its Fock wave function).

We define in this section the "interaction operator" of two parton systems, which allows to calculate the amplitude for interactions of two fast colliding hadrons. The form of this operator has to meet the requirement of correspondence with calculations according to the RFT rules.

Let two hadrons $h$ and $\tilde{h}$ move one toward another with rapidities $y$ and $\tilde{y}$ in some Lorentz frame at impact parameter $\mathbf{b}$. The essential feature required of the interaction operator is that Lorentz invariance of the scattering amplitude, that is, its independence from a choice of particular Lorentz frame, should be maintained. In other words, the amplitude should be a function of the total rapidity $Y=y+\tilde{y}$ only $f$, but not of the individual values of $y$ and $\tilde{y}$.

The hadron states are specified by sets of parton distributions $\left\{f_{s}\left(y ; \mathcal{Z}_{s}\right)\right\}$ and $\left\{\tilde{f}_{s}\left(\tilde{y} ; \tilde{\mathcal{Z}}_{s}+\mathbf{b}\right)\right\}$, where designation $\tilde{\mathcal{Z}}_{s}+\mathbf{b}$ means a shift of all coordinates $\tilde{\mathbf{z}}_{a}$ to the vector $\mathbf{b}$ in the impact plane. Denote for brevity these sets as vectors in space of states $|\mathcal{F}(y ; 0)\rangle$ and $|\tilde{\mathcal{F}}(\tilde{y} ; \mathbf{b})\rangle$. In the momentum representation we shall use notation $|\mathcal{G}(y ; 0)\rangle$ and $|\tilde{\mathcal{G}}(\tilde{y} ; \mathbf{b})\rangle$ for the sets $\left\{g_{s}\left(y ; \mathcal{Q}_{s}\right)\right\}$ and $\left\{\tilde{g}_{s}\left(\tilde{y} ; \mathcal{Q}_{s}\right) \exp \left(i \mathbf{b} \sum_{1}^{s} \mathbf{q}_{a}\right)\right\}$.

Let the total cross section is determined as an integral of function $T(y, \tilde{y} ; b)$ over all impact parameter values:

$$
\sigma^{(t o t)}(y, \tilde{y})=2 \int d^{2} b T(y, \tilde{y} ; b)
$$

$f$ Though the hadrons move in the opposite directions, we define both $y$ and $\tilde{y}$ as positive. So the total rapidity which is the rapidity value of one hadron in the rest frame of other is written as a sum $Y=y+\tilde{y}$. 
We introduce the "interaction operator" $\hat{T}$ by the following equation:

$$
\begin{aligned}
& T(y, \tilde{y} ; b)=\langle\tilde{\mathcal{F}}(\tilde{y} ; \mathbf{b})|\hat{T}| \mathcal{F}(y ; 0)\rangle= \\
& \quad=\sum_{s=1}^{\infty} \frac{(-1)^{s-1} \epsilon^{s}}{s !} \int d \tilde{\mathcal{Z}}_{s} d \mathcal{Z}_{s} \tilde{f}_{s}\left(\tilde{y} ; \tilde{\mathcal{Z}}_{s}\right) f_{s}\left(y ; \mathcal{Z}_{s}\right) \delta^{(s)}\left(\mathcal{Z}_{s}-\tilde{\mathcal{Z}}_{s}-\mathbf{b}\right)
\end{aligned}
$$

or, in momentum space,

$$
\begin{aligned}
T(y, \tilde{y} ; b) & =\langle\tilde{\mathcal{G}}(\tilde{y} ; \mathbf{b})|\hat{T}| \mathcal{G}(y ; 0)\rangle= \\
& =\sum_{s=1}^{\infty} \frac{(-1)^{s-1} \epsilon^{s}}{s !} \int \frac{d \mathcal{Q}_{s}}{(2 \pi)^{2 s}} e^{i \mathbf{b} \sum_{1}^{s} \mathbf{q}_{a}} \tilde{g}_{s}\left(\tilde{y} ; \mathcal{Q}_{s}\right) g_{s}\left(y ; \mathcal{Q}_{s}\right)
\end{aligned}
$$

The parameter $\epsilon$ plays a role of the squared size of a parton. As we mentioned in sect. 3 in order to regularize some singular expressions the function $\epsilon^{s} \delta^{(s)}$ should be smeared out over this range.

Let us show that the definition (56), (57) of the operator $\hat{T}$ combined with particular relation between constants provides the dependence of the function $T(y, \tilde{y} ; b)$ only from the single variable $Y=y+\tilde{y}$. In the momentum representation the evolution equations (45) can be symbolically written as

$$
\frac{d}{d y}|\mathcal{G}(y ; \mathbf{b})\rangle=\hat{H}|\mathcal{G}(y ; \mathbf{b})\rangle
$$

where the form of the operator $\hat{H}$ follows from eq.445). Its solution is

$$
|\mathcal{G}(y ; \mathbf{b})\rangle=\exp [\hat{H} y]|\mathcal{G}(0 ; \mathbf{b})\rangle .
$$

i.e. the evolution operator exp $[\hat{H} y]$ has a meaning of the Lorentz boost operator. Therefore

$$
T(y, \tilde{y} ; b)=\left\langle\mathcal{G}(0 ; b)\left|e^{\hat{H}^{t} \tilde{y}} \hat{T} e^{\hat{H} y}\right| G(0 ; 0)\right\rangle,
$$

( $\hat{H}^{t}$ is the transpose of $\hat{H}$ ). If the relation

$$
\left\langle\chi\left|\hat{H}^{t} \hat{T}\right| \phi\right\rangle=\langle\chi|\hat{T} \hat{H}| \phi\rangle
$$

holds for arbitrary states $|\phi\rangle, \mid \chi>$, then the required property will be fulfilled:

$$
\left\langle\chi\left|e^{\hat{H}^{t} \tilde{y}} \hat{T} e^{\hat{H} y}\right| \phi\right\rangle=\left\langle\chi\left|\hat{T} e^{\hat{H}(y+\tilde{y})}\right| \phi\right\rangle .
$$


Let us prove the relation (61). The first, second, and fourth terms of the matrix $\hat{H}$ (see eq. (45)) are diagonal in the parton numbers $s$, and the relation (61) is evident. The third and fifth terms (denote them as $H_{12}$ and $H_{21}$ ) correspond to splitting and fusion of reggeons and their matrix elements have to be conjugated to each other, i.e. $\left\langle\chi\left|T H_{21}\right| \phi\right\rangle=\left\langle\chi\left|H_{12}^{t} T\right| \phi\right\rangle$ and vice versa. Indeed, the matrix element of $T H_{21}$ is written as

$$
\begin{aligned}
\left\langle\chi\left|T H_{21}\right| \phi\right\rangle= & -\left(2 m_{2}+\nu\right) \sum_{s=1}^{\infty} \frac{(-1)^{s-1} \epsilon^{s}}{s !} \int \frac{d \mathcal{Q}_{s}}{(2 \pi)^{2 s}} \frac{d \mathbf{q}}{(2 \pi)^{2}} \chi_{s}\left(\mathcal{Q}_{s}\right) \\
& \times \sum_{k=1}^{s} \phi_{s+1}\left(\mathcal{Q}_{s}^{(k)}, \mathbf{q}_{k}+\mathbf{q},-\mathbf{q}\right)=\left(2 m_{2}+\nu\right) \sum_{s=1}^{\infty} \frac{(-1)^{s} \epsilon^{s}}{(s-1) !} \\
& \times \int \frac{d \mathcal{Q}_{s}}{(2 \pi)^{2 s}} \frac{d \mathbf{q}}{(2 \pi)^{2}} \chi_{s}\left(\mathcal{Q}_{s}\right) \phi_{s+1}\left(\mathcal{Q}_{s}^{(k)}, \mathbf{q}_{k}+\mathbf{q},-\mathbf{q}\right),
\end{aligned}
$$

with regard that the sum over $k$ contains $s$ identical terms. In the matrix element of $T_{12}$ summation over parton number starts with $\tilde{s}=2$. Hence, after substitution $\tilde{s}=s+1$ and taking into account that the sum over $k, l$ contains $s(s+1)$ identical terms one gets

$$
\begin{aligned}
\left\langle\chi\left|H_{12}^{t} T\right| \phi\right\rangle= & \left\langle\phi\left|T H_{12}\right| \chi\right\rangle=\lambda \sum_{\tilde{s}=2}^{\infty} \frac{(-1)^{\tilde{s}-1} \epsilon^{\tilde{s}}}{\tilde{s} !} \int \frac{d \mathcal{Q}_{\tilde{s}}}{(2 \pi)^{2 \tilde{s}}} \phi_{\tilde{s}}\left(\mathcal{Q}_{\tilde{s}}\right) \\
& \times \sum_{k, l=1}^{\tilde{s}} \chi_{\tilde{s}-1}\left(\mathcal{Q}_{\tilde{s}}^{(k l)}, \mathbf{q}_{k}+\mathbf{q}_{l}\right)=\lambda \epsilon \sum_{s=1}^{\infty} \frac{(-1)^{s} \epsilon^{s}}{(s-1) !} \\
& \times \int \frac{d \mathcal{Q}_{s+1}}{(2 \pi)^{2(s+1)}} \chi_{s}\left(\mathcal{Q}_{s-1}, \mathbf{q}_{s}+\mathbf{q}_{s+1}\right) \phi_{s+1}\left(\mathcal{Q}_{s+1}\right) .
\end{aligned}
$$

After change of integration variables the both matrix elements are the same provided

$$
2 m_{2}+\nu=\lambda \epsilon
$$

In the case of the 0 -dimensional model constants $\lambda, m_{2}$ and $\nu$ are dimensionless, the parameter $\epsilon$ in the definition of the interaction operator is not needed and the requirement of Lorentz invariance gives the relation

$$
2 m_{2}+\nu=\lambda .
$$

The conditions (65), (66) look quite natural from the point of view of RFT (equality of three-pomeron vertices). 


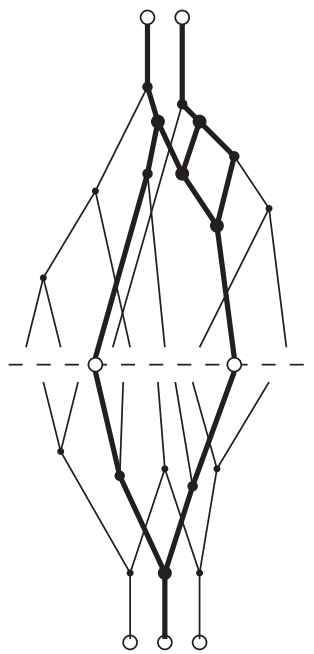

(a)

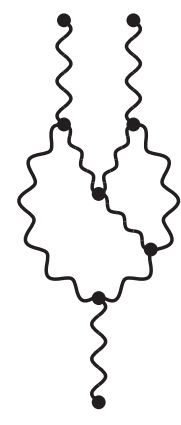

(b)

Figure 2: Correspondence between parton cascade history and reggeon diagrams

Let us discuss briefly relation between diagrams representing a history of development of parton cascades and the RFT diagrams. Fig.2a gives an example of evolution of parton distributions for the colliding hadrons with rapidity. In this example two partons from each hadron are linked in some intermediate Lorentz frame $(s=2$ in eqs (56)), (57)). The cascade branches with "dead ends", i.e. terminated in noninteracting partons (thin lines in fig.2a), result in renormalization of distribution functions (see sect.4) after summation over all possibilities of the "dead-end" branches. The renormalized propagators are showed by thick lines at the figure and form a skeleton of the parton network. Each skeleton corresponds to a particular order of reggeon perturbation theory in number of splittings and fusions of renormalized reggeon propagators. Different ways of linkage of partons from interacting hadrons give different skeletons, i.e. different reggeon diagrams. Skeleton can be imaginated pictorially as a set of lines in the cascade network with non-zero electric current flow if to ascribe definite potentials to the sets of initial points of the projectile and target.

Note that the interaction operator (56) doesn't have a sense of probability of interaction for two parton systems because it takes into account only single interactions of partons. Similar situation one can see in descrimtion of nucleus-nucleus scattering in so called Czyź-Maximon approximation 14 which 
includes only first-order interactions of every nucleon. It describes correctly the scattering amplitude only for low nuclear densities, and account for all orders of nucleon interactions is necessary to get unitary amplitude for high densities. However, in case of parton model, introduction of higher order terms into interaction operator would violate Lorentz invariance of the amplitude.

\section{Examples}

\subsection{Eikonal approximation}

Consider first hadron scattering in the model including parton diffusion but without any parton transformations $\left(m_{1}=m_{2}=\lambda=\nu=0\right)$. In this approximation each parton walks randomly and independently in the transverse plane, therefore, if there were no correlations in the initial parton distributions for both hadrons there will be no correlations in the course of further evolution:

$$
f_{s}\left(y ; \mathcal{Z}_{s}\right)=\prod_{a=1}^{s} f_{1}\left(y ; z_{a}\right), \quad \tilde{f}_{s}\left(\tilde{y} ; \tilde{\mathcal{Z}}_{s}\right)=\prod_{a=1}^{s} \tilde{f}_{1}\left(\tilde{y} ; \tilde{z}_{a}\right) .
$$

The amplitude of hadron interaction at the impact parameter $b$ is determined by eq.(56) and coincides with the eikonal approximation formula (see fig.3a)

$$
T^{(e i k)}(Y, b)=\sum_{s=1}^{\infty} \frac{(-1)^{s-1}}{s !} \chi(Y, b)=1-e^{-\chi(Y, b)},
$$

where $Y=y+\tilde{y}$, and so called eikonal function is equal to

$$
\chi(Y, b)=\epsilon \int d \mathbf{z} f_{1}(y, \mathbf{z}) \tilde{f}_{1}(\tilde{y}, \mathbf{z}-\mathbf{b})=\epsilon \int \frac{d \mathbf{q}}{(2 \pi)^{2}} e^{i \mathbf{q b}} g(y, \mathbf{q}) \tilde{g}(\tilde{y}, \mathbf{q}) .
$$

If the initial distributions in hadrons were of the $\delta$-function type, then one-particle distribution at finite $y$ is determined by random walk only:

$$
f_{1}(y, z)=\frac{1}{4 \pi y D} e^{-\frac{z^{2}}{4 y D}}, \quad g_{1}(y, q)=e^{-y D q^{2}},
$$

and similarly for the hadron $\tilde{h}$. This gives

$$
\chi(Y, b)=\frac{\epsilon}{4 \pi Y D} e^{-\frac{b^{2}}{4 Y D}} .
$$

If the initial distribution is given by some function $r(z)$ with Fourier transform

$$
s(q)=\int d \mathbf{z} e^{i \mathbf{q} \mathbf{z}} r(z),
$$




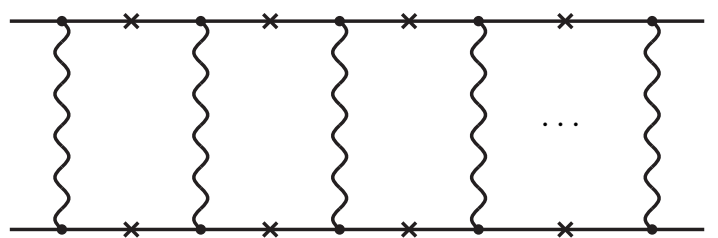

(a)

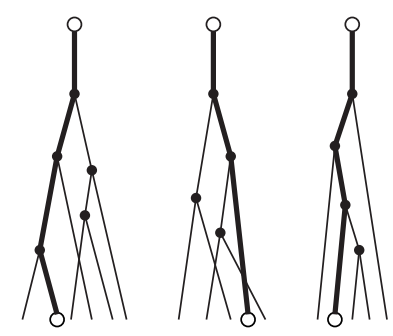

(b)

Figure 3: (a) Reggeon diagrams corresponding to the eikonal approximation; (b) Parton interpretation of the eikonal approximation for supercritical pomeron

then the eikonal is determined by formula

$$
\chi(Y, b)=\epsilon \int \frac{d \mathbf{q}}{(2 \pi)^{2}} e^{i \mathbf{q b}} s(q) \tilde{s}(q) e^{-Y D \mathbf{q}^{2}} .
$$

It is seen that eq.(73) corresponds to exchange of the regge pole with intercept equaled to 1 , trajectory slope $\alpha^{\prime}=D$ and residue $s(q) \tilde{s}(q)$.

Often the formulae of the eikonal approximation are applied in the case of supercritical pomeron with intercept larger than 1 . It seems to be inconsistent in the model under discussion because in the parton picture the supercritical regime means the growth of parton number when rapidity changes and this can occur only due to parton splitting in the course of evolution, i.e. at nonzero value of the constant $\lambda$. The eikonal approximation in the presence of splitting means that among soft partons originated due to evolution of each initial partons only one may interact with a target (see fig.3b). The more consistent consideration of parton splitting allowing interaction with the target for any parton is carried out in the next example. However, the analytic formulae can be obtained only if parton diffusion is disregarded.

\subsection{The 0-dimensional Schwimmer model}

The Schwimmer modell was developed for description of inelastic interaction of fast hadron with heavy nucleus and corresponds to summing enhanced reggeon diagrams in the tree approximation (see fig.4). If a radius of the nucleus is large enough $\left(R_{A} \gg \alpha^{\prime} Y\right)$, then parton diffusion can be disregarded and the problem is equivalent to the 0 -dimensional model of sect.2a (we assume that $\left.\lambda \neq 0, m_{1} \neq 0, m_{2}=\nu=0\right)$.

Choose the initial state of the projectile hadron as containing one parton with 


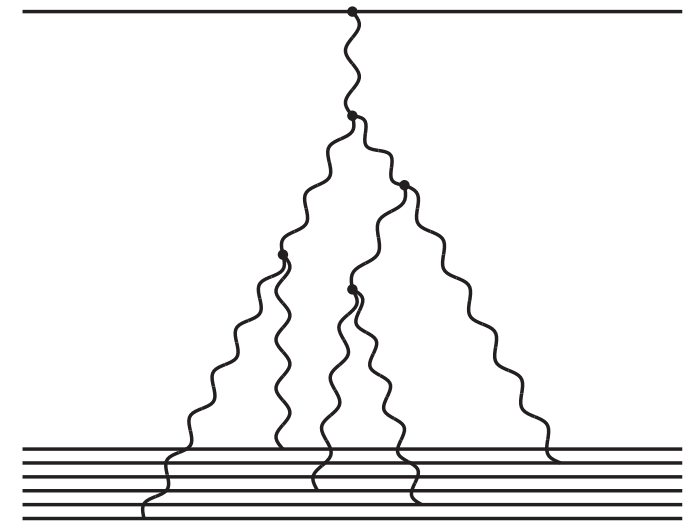

Figure 4: Reggeon diagrams corresponding to the Schwimmer approximation

a probability $g_{p}$

$$
\begin{aligned}
& p_{1}^{(p)}=g_{p}, \quad p_{0}^{(p)}=1-g_{p}, \quad p_{N}^{(p)}=0 \quad \text { at } \quad N \geq 2, \\
& G^{(p)}(w, 0)=1+g_{p}(w-1)
\end{aligned}
$$

and the initial parton distribution for the target in the Poisson form

$$
p_{N}^{(t)}(0)=\frac{g_{t}^{N}}{N !} e^{-g_{t}}, \quad G^{(t)}(w, 0)=e^{g_{t}(w-1)}, \quad \mu_{s}^{(t)}(0)=\left(g_{t}\right)^{s} .
$$

The fusion is absent in this model, $\nu=0$, so the Lorentz-invariant consideration is impossible. The interaction amplitude calculated in the laboratory frame has according to the 0 -dimensional version of the formula (56) the following form:

$$
\begin{aligned}
T_{s c h w}(Y) & =\sum_{s=1}^{\infty} \frac{(-1)^{s-1}}{s !} \mu_{s}^{(p)}(Y) \mu_{s}^{(t)}(0) \\
& =1-G^{(p)}\left(1-g_{t} ; Y\right)=\frac{g_{p} g_{t} e^{\Delta Y}}{1+\frac{g_{t} \lambda}{\Delta}\left(e^{\Delta Y}-1\right)} .
\end{aligned}
$$

We used the definition (5) for the generating function $G^{(p)}$ and its explicit form (14), (15) as applied to the initial condition (74): $G^{(p)}(w ; Y)=g_{p} W(Y)$, with $W(Y)$ from eq.(15). 
When one estimates the cross section of hadron interaction with a nucleus $A$ one has to choose $g_{t}$ to be proportional to the nuclear density $\rho_{A}(\mathbf{b})$ :

$$
g_{t}=g_{N} \rho_{A}(\mathbf{b}) .
$$

For heavy nucleus $(A \gg 1)$ in the approximation of constant density,

$$
\rho_{A}=\frac{A}{\pi R_{A}^{2}} \theta\left(R_{A}-|\mathbf{b}|\right), \quad R_{A}=r_{0} A^{1 / 3},
$$

we come to

$$
T_{A}(Y, b)=\frac{g_{p} g_{N} A^{1 / 3}}{\pi r_{0}^{2}} \frac{\exp (\Delta Y) \theta\left(R_{A}-|\mathbf{b}|\right)}{1+\frac{\lambda g_{N} A^{1 / 3}}{r_{0}^{2}}\left(\frac{\exp (\Delta Y)-1}{\Delta}\right)}
$$

This formula coincides with the original Schwimmer formula for amplitude of hadron-nucleus scattering in the approximation of very large nucleus. When $Y \rightarrow \infty$ the amplitude goes to the constant limit $g_{p} \Delta / \lambda=g_{p}\left(1-m_{1} / \lambda\right)<1$. Note that in general reggeon field theory the constants $g_{p}, \Delta$ and $\lambda$ may be arbitrary.

It is worth to stress that though the formula $(79)$ coincides in form with the Schwimmer formula obtained in RFT, the meaning of the constants entering these formulae is different, and even their dimensions differ: in eq. (79) the constants $g_{p}$ and $\lambda$ are dimensionless, and $g_{N}$ has dimension of length squared, while in reggeon field theory all these constants have dimension of length.

It is easy to formulate a generalization of the Schwimmer approximation in this approach. If the initial parton distribution in the projectile has the Poisson form, i.e.

$$
\mu_{s}^{(p)}(0)=\left(g_{p}\right)^{s}, \quad G^{(p)}(w, 0)=\exp \left[g_{p} w\right], \quad G^{(p)}(w, Y)=\exp \left[g_{p} \tilde{w}(Y)\right],
$$

one gets the "eikonalized" Schwimmer formula

$$
T_{\text {schw }}^{(e i k)}(Y)=1-\exp \left[T_{s c h w}(Y)\right] .
$$

Note that analysis of interaction of two heavy nuclei requires even in the tree approximation taking into consideration not only parton splitting but their fusion also. Moreover, as it was emphasized already, the Lorentz-invariant parton consideration is possible only in presence both splitting and fusion of partons provided the corresponding constants are related. 


\subsection{Asymptotics of the 0-dimensional model with account for parton fusion}

We consider in this section the 0-dimensional model including splitting and fusion of partons for the case $m_{1}=m_{2}=0$. Let us demonstrate the Lorentz invariance of the parton picture for asymptotically large rapidity value $Y$, when a stationary regime sets in and the interaction amplitude does not depend on $Y$ at all. Indeed, in this case for any frame choice one of hadron rapidities (say, $y$ ) is large. Hence the projectile hadron is in asymptotical stationary state (see sect.2.b)

$$
\mu_{s}^{(p)}(y)=B^{(p)} a^{s}, \quad B^{(p)}=\frac{1-G_{0}^{(p)}(0)}{1-e^{-a}}, \quad a=\lambda / \nu=1,
$$

and the state of a target with rapidity $\tilde{y}$ is given by the generating function $G^{(t)}(\tilde{y}, w)$. It is essential that the value of this function at $w=0$ does not depend on $\tilde{y}$ and is equal to $G_{0}^{(t)}(0)$.

Therefore for $a=1$ the interaction amplitude does not depend on rapidity in any Lorentz frame and equals

$$
\begin{aligned}
T_{\infty}(Y)=\sum_{s=1}^{\infty} \frac{(-1)^{s-1}}{s !} \mu_{s}^{(p)}(y) \mu_{s}^{(t)}(\tilde{y}) & =B^{(p)}\left[1-G^{(t)}(\tilde{y}, 1-a)\right] \\
& =\frac{\left[1-G_{0}^{(p)}(0)\right]\left[1-G_{0}^{(t)}(0)\right]}{1-e^{-1}}
\end{aligned}
$$

\section{Two-dimensional diffusion}

In presence of parton diffusion it is possible to distinguish in the parton structure of the fast hadron two characteristic regions. At the periphery where parton density is small the parton fusion is insignificant and only diffusion and splitting processes occur. Parton distribution in this region behaves like the Green function of diffusion equation complemented with exponential increase due to splitting:

$$
f_{1}^{(\text {periph })}(y, z)=e^{\lambda y} \frac{1}{4 \pi y} e^{-z^{2} / 4 D y} .
$$

In the central region the parton fusion is essential and the density comes to the stationary value

$$
f_{1}^{(\text {centr })}(y, z) \approx \lambda / \nu
$$


Transition from one regime to another corresponds to equality of the central and peripheral density values, i.e. to distances of order

$$
R(y) \approx 2 \sqrt{\lambda D} y
$$

At larger distances the density decreases exponentially. Thus, the fast hadron represents to be a disc of constant density of radius increasing proportionally to its rapidity:

$$
f_{1}(y, z) \simeq \frac{\lambda}{\nu} \theta[R(y)-z] .
$$

Obviously the interaction cross section of two such discs corresponds to the Froissart regime: $\sigma(Y) \approx$ const $\cdot Y^{2}$, where $Y=y+\tilde{y}$. In order to calculate the constant value, i.e. the degree of blackness, it is necessary to account for contributions of all multi-particle densities $f_{s}\left(y, \mathcal{Z}_{s}\right), \tilde{f}_{s}\left(\tilde{y}, \mathcal{Z}_{s}\right)$. It is not the aim of this paper to carry out complete analysis of this problem. Let us consider as an illustration only the simplest case when the initial state of one of hadrons (say, $\tilde{h}$ ) contains only one parton, that is $\tilde{f}_{1}(\tilde{y}=0, z)=\delta(b-z)$, $\tilde{f}_{s}\left(\tilde{y}=0, \mathcal{Z}_{s}\right)=0, s \geq 2$. Let us use the Lorentz invariance of the problem and apply the formula $(56)$ in the rest frame of the hadron $\tilde{h}$. Then in the formula only the first term remains:

$$
\begin{aligned}
T(y, \tilde{y} ; b) & =\epsilon \int d z f_{1}(Y ; z) \tilde{f}_{1}(0 ; \tilde{z}) \delta(\mathbf{z}-\tilde{\mathbf{z}}-\mathbf{b}) \\
& \approx \frac{\epsilon \lambda}{\nu} \theta(R(Y)-b)=\theta(R(Y)-b),
\end{aligned}
$$

which corresponds due to relation (65) to scattering on the absolutely black disk of radius $R(Y)$.

For more complicated cases (many-parton initial states) the situations turnes out to be the same - the asymtotics of the amplitude corresponds to the scattering off a black disc. In ref. 15 Kancheli discussed the problems related to the Lorentz invariance of interaction of parton systems for the case of the gray disc (with finite transparency). In present model this problem does not appear, and both Lorentz invariance and blackness of the amplitude are provided by relation (65).

\section{Unitarity}

One of the key problems of RFT is s-channel unitarity of the scattering amplitude. As can be seen, the unitarity of the amplitude calculated for the model 
under consideration depends not only from the parton dynamics but also from the initial conditions, i.e. from the form of the parton sources for each hadron.

Let us discuss under which conditions the amplitude $T(Y, b)$ can be interpreted as a probability of interaction at fixed impact parameter that is satisfies to the relation

$$
0<T(Y, b)<1 .
$$

Consider first the case with $d=0$ and note that if both sets of moments $\mu_{s}(y), \tilde{\mu}_{s}(Y-y)$ in equation

$$
T(Y, b)=\sum_{s=1}^{\infty} \frac{(-1)^{s-1}}{s !} \mu_{s}(y) \tilde{\mu}_{s}(Y-y)
$$

would be of Poisson form,

$$
\mu_{s}=\alpha^{s}, \quad \tilde{\mu}_{s}=\tilde{\alpha}^{s},
$$

the amplitude has a simple form satisfying to relations (89):

$$
T^{(\alpha, \tilde{\alpha})}(Y, b)=1-\exp (\alpha \tilde{\alpha}) .
$$

This observation makes useful so called Poisson representation 16 for probabilities. It represents (for the simplest case) probabilities $p_{N}(y)$ as superpositions of Poisson distributions $P_{N}(\alpha)$ :

$$
p_{N}(y)=\int d \alpha f(\alpha, y)\left\{\frac{\alpha^{N}}{N !} e^{-\alpha}\right\}=\left\langle P_{N}(\alpha)\right\rangle,
$$

where we used notation $\langle\ldots\rangle$ for averaging with weight $f(\alpha, y)$.

The generating function $G(w ; y)$ can be written as

$$
G(w ; y)=\int d \alpha f(\alpha, y) \exp [(w-1) \alpha] .
$$

The master equation (6) gives for $f(\alpha, y)$ the equation of Fokker-Planck type

$$
\frac{\partial f(\alpha, y)}{\partial y}=-\frac{\partial}{\partial \alpha}\{A(\alpha)\}+\frac{1}{2} \frac{\partial^{2}}{\partial \alpha^{2}}\{B(\alpha)\} .
$$

where functions

$$
A(\alpha)=\left(\lambda-m_{1}\right) \alpha-\left(\nu+2 m_{2}\right) \alpha^{2}, \quad B(\alpha)=2 \lambda \alpha-2\left(\nu+m_{2}\right) \alpha^{2},
$$


describe driving term and diffusion coefficient correspondingly. The diffusion coefficient $B$ is non-negative for $0 \leq \alpha \leq \lambda /\left(\nu+m_{2}\right)$. The condition (8) provides normalization of $f(\alpha, y)$ :

$$
\int d \alpha f(\alpha, y)=1
$$

Equation (95) is equivalent 16 to the differential stochastic equation

$$
d \alpha=A(\alpha) d y+\sqrt{B(\alpha)} d W(y),
$$

where $d W(y)$ is a stochastic differential corresponding to the Wiener process.

Suppose for simplicity that $m_{1}=m_{2}=0$. One can see from (98) that if evolution starts at $y=0$ at $\alpha$ value within interval $(0, a), a=\lambda / \nu$, then the first term will drive the system to the right end of the interval $a=\lambda / \nu)$. At this point both the driving term and diffusion coefficient vanish and, therefore, it is a stationary point. Any initial $\alpha$-distribution will be concentrated at this point when $y \rightarrow \infty$. Thus the asymptotic distribution is Poisson distribution $P_{N}(a)$, in correspondence with analysis of section 2 .

Because of positivity of the diffusion coefficient $B(\alpha)$ at the interval $(0, a)$, the distribution $f(\alpha, y)$ is positive and normalizable in the course of evolution, i.e. it can be considered as a probability distribution. If both $f(\alpha, y)$ and $\tilde{f}(\alpha, \tilde{y})$ are of class of probability distributions, then the interaction amplitude $T(Y, b)$ is given by averaging of equation (92) over $\alpha$ and $\tilde{\alpha}$,

$$
T(Y, b)=\left\langle\left\langle T^{(\alpha, \tilde{\alpha})}(Y, b)\right\rangle\right\rangle \equiv \int d \alpha f(\alpha, y) \int d \tilde{\alpha} f(\tilde{\alpha}, \tilde{y})[1-\exp (\alpha \tilde{\alpha})],
$$

and evidently satisfies the unitarity condition (89). Consideration of twodimensional case can be carried out similarly.

\section{Conclusions}

The parton stochastic model discussed in this paper is in exact correspondence with reggeon field theory. Only special reggeon field theories with particular set of pomeron vertices allow parton interpretation of this sort. The model reproduces results of main approximations for RFT. In the theory with zero number of transverse dimensions, $d=0$, it is possible to analyze quantitatively an asymptotical regime. It is equivalent to summing all diagrams of 0 -dimensional RFT with all loops. For theory with $d=2$ qualitative and numerical consideration is possible. Because of its classical and stochastic nature the model allows numerical simulation by means of simple Monte 
Carlo algorithm. Direct analogy with kinetic theory of chemical reactions gives good prospects for application of thermodynamical methods and of theory of stochastic equations.

The model allows a number of generalizations, in particular to parton systems with internal quantum numbers and of various spacial scales.

\section{Acknowledgments}

The author is thankful to A.B. Kaidalov and O.V. Kancheli for numerous stimulating discussions and to M.A. Braun, Yu.L. Dokshitzer and Yu.A. Simonov for discussion of results. This work has been funded in part by the Russian Foundation of Basic Researches (RFBR 98-02-17463, 00-15-9678), by the NATO grant PSTCLG 977275 and by the INTAS grant $00-00366$.

\section{References}

1. "Regge Theory of Low- $p_{T}$ Hadronic Interactions", ed. by L. Caneschi, Elsevier Science Publishers B.V., 1989.

2. V.N. Gribov, Zh. Exp. Theor. Fiz. 53, 654 (1967); [Sov. Phys. JETP 26, 414 (1968)].

3. D. Amati, A. Stanghellini, and S. Fubini, Nuovo Cimento 26, 896 (1962).

4. S. Mandelstam, Nuovo Cimento 30, 1113; 1127; 1143 (1963).

5. A. Kaidalov, in " $Q C D$ at $200 \mathrm{TeV}$ ", ed. L. Cifarelli and Yu. Dokshitzer, Plenum Press (1992), p. 1.

6. L.N. Lipatov, Sov. J. Nucl. Phys. 23, 338 (1976); E.A. Kuraev, L.N. Lipatov, V.S. Fadin, Sov. Phys. JETP 45, 199 (1977); Y.Y. Balitsky, L.N. Lipatov, Sov. J. Nucl. Phys. 28, 822 (1978).

7. A. Schwimmer, Nucl. Phys. B 94, 445 (1975)

8. J.L. Cardy, Nucl. Phys. B 75, 413 (1974)

9. M.S. Dubovikov, K.A. Ter-Martirosyan, Nucl. Phys. B 124, 163 (1977)

10. A.B. Kaidalov, L.A. Ponomarev, K.A. Ter-Martirosyan, Yad. Fiz. 44, 722 (1986); [Sov. J. Nucl. Phys. 44, 468 (1986)].

11. P. Grassberger, K. Sundermeyer, Phys. Lett. B 72, 220 (1978)

12. V.A. Abramovskii, V.N. Gribov, O.V. Kancheli, Yad. Fiz. 18, 595 (1973); [Sov. J. Nucl. Phys. 18, 308 (1974)].

13. K.G. Boreskov, A.B. Kaidalov, Acta Phys. Pol. B 20, 397 (1989);

14. W. Czyź, L.C. Maximon, Ann. Phys. 52, 59 (1969)

15. O. Kancheli, "About the structure of the Froissart limit in QCD", to be published, hep-ph/0008299.

16. C.W. Gardiner, "Handbook of stochastic methods for Physics, Chemistry and the Natural Sciences", (Springer-Verlag, Berlin, Heidelberg 1985) 